\title{
Appraisal of vitreous syphilis antibody as a novel biomarker for the diagnosis of syphilitic uveitis: a prospective case-control
} \section{study}

\author{
Sukhum Silpa-archa $\mathbb{D}^{1 凶}$, Tararat Hoopholerb ${ }^{1}$ and Charles Stephen Foster $\mathbb{D}^{2,3,4}$ \\ (c) The Author(s), under exclusive licence to The Royal College of Ophthalmologists 2021
}

\begin{abstract}
PURPOSE: To determine the sensitivity and specificity of syphilis antibody tests in vitreous samples and to propose an algorithm using vitreous syphilis antibody as a supplementary test to confirm syphilitic uveitis (SU).

METHODS: A prospective case-control study was conducted at the Retina and Uveitis Clinic from May 2017 to January 2020. Initially, patients were classified based on syphilis serology into group 1 (positive testing) and group 2 (negative testing). Group 1 was further divided into 2 subgroups (group 1A and 1B) depending on their relevant clinical manifestations and clinical improvement. Group 2 served as a control group.

RESULTS: Thirty-eight patients were enrolled in the study: 14 in group 1A, 5 in group 1B, and 19 in group 2B. No patient was assigned to group $2 \mathrm{~A}$. All patients in group $1 \mathrm{~A}$, representing definite $\mathrm{SU}$, completed syphilis test (rapid plasma reagin [RPR], enzyme immunoassay [EIA], and fluorescent treponemal antibody-absorption [FTA-ABS]) for vitreous, and all vitreous samples yielded positive results. Of the 5 subjects in group 1B, 3 cases were considered to be not SU with different conditions, and 2 were indeterminate for SU. They presented with different features not typical of SU, and they had variable and fewer positive syphilis antibody responses. The most sensitive test for detecting syphilis antibodies in vitreous was EIA (90.9\%), followed by RPR (80.0\%) and FTA-ABS lgG (78.9\%). EIA and FTA-ABS had the highest specificity, detecting $100 \%$ of the syphilis antibody.

CONCLUSIONS: Vitreous analysis of syphilis antibody can serve as a supplementary test to confirm SU in selected cases as the proposed algorithm.
\end{abstract}

Eye (2023) 37:146-154; https://doi.org/10.1038/s41433-021-01902-6

\section{INTRODUCTION}

Presumptive diagnosis of syphilitic uveitis (SU) in uveitis patients is made with positive serology results from a nontreponemal test such as the rapid plasma reagin (RPR), and it is then followed by confirmation using one of the treponemal tests [1-3]. However, many researchers include active uveitis patients with only positive treponemal serologic test in their cohort [3-6]. In most cases of SU, serological diagnosis and clinical presentations are sufficient to make a presumptive diagnosis. Nevertheless, besides unusual serologic responses in HIVinfected patients as reported [7-11], problems with diagnosis and treatment may occur in some uveitis patients if they have discordant or equivocal syphilis serology [1, 12, 13], atypical clinical presentation [14-16], or, in immunocompromised patients, possible co-infection [15, 17]. Given the unavailability of Treponema pallidum polymerase chain reaction (PCR) in many areas, only a few cases diagnosed by PCR analysis for T. pallidum in vitreous samples have been reported in the literature $[15,18,19]$. Besides, newer PCR-based techniques have low sensitivity in detecting syphilis in blood $[20,21]$ and cerebrospinal fluid (CSF) [22], and thus, they cannot replace the abovementioned serological tests.
The novel application of nontreponemal and treponemal antibody to confirm diagnosis of SU from vitreous samples was recently demonstrated by the current authors [14]. Therefore, the purpose of this study was not only to determine the sensitivity and specificity of nontreponemal and treponemal antibody testing in vitreous samples but also to propose an algorithm using vitreous syphilis antibody to serve as a supplementary test to serology for the confirmation of syphilitic retinitis/ chorioretinitis.

\section{METHODS}

\section{Study design and population}

This study was a prospective case-control study conducted at the Retina and Uveitis Clinic, Department of Ophthalmology, Rajavithi Hospital, Bangkok, Thailand. The study followed the tenets of the Declaration of Helsinki and was approved by the Ethics Committee of Rajavithi Hospital (approval No. 119/2561). Patients giving informed consent for participation were enrolled from January 2017 to January 2020. Diagnosis of SU was confirmed using positive serologic tests, including a nonspecific test (RPR), and one of the specific treponemal antibody tests, i.e., enzyme immunoassay (EIA), the fluorescent treponemal antibody-absorption (FTA-ABS) immunoglobulin M (IgM), or immunoglobulin G (lgG). Analyses


MA, USA. ${ }^{3}$ Ocular Immunology \& Uveitis Foundation, Waltham, MA, USA. ${ }^{4}$ Harvard Medical School, Boston, MA, USA. ${ }^{\circledR}$ email: sukhumsilp@gmail.com 
of each serum sample using RPR (Agappe, Kerala, India), EIA (DIESSE, Monteriggioni (Siena), Italy) and FTA-ABS (EUROIMMUN, Lubeck, Germany) were performed according to the manufacturers' instructions.

A negative serologic test for syphilis was defined as negative results both from RPR and from one of the specific treponemal antibody tests (EIA, FTA-ABS lgG). Patients with other immunological or infectious systemic diseases except human immunodeficiency virus (HIV) infection were excluded. A group of non-infectious uveitis patients who tested negative for syphilis in serological tests and had no symptoms or signs of active syphilis served as the controls for experiments on vitreous specimens. The required sample size was calculated and the calculated required minimum sample size was set at not less than 32 (See Supplementary information 1 for sample size calculation). Finally, 38 cases were enrolled and initially categorized into 2 arms based on results of the serum syphilis antibodies: positive and negative.

\section{Data collection}

Baseline demographics and past medical history were retrieved. Ophthalmological findings included best-corrected visual acuity (BCVA), intraocular pressure measurement, slit-lamp examination findings, type of intraocular inflammation, grade of inflammation in the anterior chamber and in the vitreous, and fundus examination. Criteria from the Standardization of Uveitis Nomenclature Working Group were used for uveitis classification and inflammation grading [23]. To confirm the diagnosis and exclude other causes of uveitis, all patients underwent a complete eye examination, laboratory examination, and imaging studies, which included chest $x$-ray, syphilis test, Anti-HIV test, and anti-Toxoplasma antibodies. When available, CSF data also were collected (routine biochemical, cytologic analyses, and syphilis testing). Color fundus photographs, spectral domain optical coherence tomography (SD-OCT) (Spectralis OCT, Heidelberg, Germany), and fluorescein angiography (FA) were performed for all patients if the fundus details were visible.

Once diagnosed with SU, patients were admitted, and neurologists and infectious disease specialists were consulted for further recommendations. Treatment for neurosyphilis was justified by an infection specialist and was administered to each patient, either 4 million units of penicillin $G$ intravenously every 4 hours or $2 \mathrm{~g}$ daily of ceftriaxone administered intravenously for 14 days [24, 25]. Treatment for syphilis was given to all cases with positive serology for syphilis. SU activity was evaluated at 1 week, 2 weeks, and 4 weeks after treatment initiation, and finally at follow-up. In addition, systemic corticosteroids were given if a patient showed no signs of improvement (visual gain, clinical manifestations, and imaging findings) at 2 weeks after the onset of antibiotic treatment.

\section{Vitreous sample processing and interpretation}

Vitreous fluid was collected by tapping or pars plana vitrectomy. Basically, the minimum vitreous volumes needed were $0.1 \mathrm{ml}$ for RPR, $0.05 \mathrm{ml}$ for EIA, and $0.05 \mathrm{ml}$ for FTA-ABS testing. However, to guarantee an adequate volume of vitreous specimen which could be depleted at times and decreased in the process of dividing the specimen, the minimal required volume was $0.3 \mathrm{ml}$ to complete all syphilis testing. Analyses of each vitreous sample were performed using the same methods for serum. In our study, vitreous syphilis positive was defined in accordance with serological diagnosis, i.e., positive RPR and either positive EIA or FTA-ABS.

\section{Patient classification}

Patients were classified based on syphilis serology into groups 1 and 2 . Group 1, with positive syphilis serology, was further divided into 2 subgroups (group $1 \mathrm{~A}$ and $1 \mathrm{~B}$ ) by evaluating the relevant clinical manifestations at initial presentation and significant clinical improvement in response to either penicillin $\mathrm{G}$ or ceftriaxone (See Supplementary information 2 for flowchart demonstrating patient classification for the study population). Clinical improvement was judged as significant when all the following criteria were met within 2-4 weeks after treatment onset: (1) visual improvement confirmed either by patients or by visual acuity measurement; (2) clinical amelioration of intraocular inflammation (a 2-step decrease of both anterior chamber and vitreous haze inflammation levels, or by the resolution of fundus lesions being either decreased vascular sheathing or size reduction in chorioretinal/retinal lesions); and (3) retinal imaging (OCT or FA) showing resolution of inflammation $[4,26]$. The controls (group 2) comprised non-infectious uveitis patients with negative syphilis serology. Their vitreous fluid was obtained in addition to the primary purpose of PCR analysis for the detection of suspected viral causes.
Group 2 was divided into 2 subgroups (group $2 \mathrm{~A}$ and $2 \mathrm{~B}$ ) based on syphilis test results from vitreous fluid. Therefore, there were 4 groups: "definite SU" group 1A-positive syphilis serology with relevant clinical features and significant clinical improvement; "not SU or indeterminate SU" group 1B positive syphilis serology with irrelevant clinical features and unclear clinical improvement in response to either penicillin $\mathrm{G}$ or ceftriaxone; group 2A-negative syphilis serology and positive vitreous syphilis antibody; and group 2B-negative syphilis serology and negative vitreous syphilis antibody.

\section{Statistical analysis}

Statistical analysis was performed using IBM SPSS Statistics for Windows, Version 20.0 (Armonk, NY: IBM Corp. 2011). The Shapiro-Wilk test was used to test for normal distribution. Characteristics of patients and eyes were reported as numbers and percentages for categoric variables and as mean or median for continuous variables.

Chi-square test and Fisher's exact test were used for univariate analysis of categorical variables. The independent-samples t-test or Mann-Whitney $U$ test were used to compare data between two unrelated groups. Sensitivity and specificity with a $95 \%$ confidence interval $(\mathrm{Cl})$ of syphilis test results from vitreous fluid were calculated using syphilis serology testing as the gold standard. Cohen's kappa coefficient was calculated to assess agreement between serum and vitreous syphilis tests. Serum and vitreous RPR titers were assessed for bivariate correlation using Spearman's test, and a $p$ value $<0.05$ was considered to be significant.

\section{RESULTS}

Our study included 19 cases (35 eyes) with positive syphilis serology and 19 controls with negative syphilis serology. Group $1 \mathrm{~A}$ (definite SU) consisted of a total of 27 eyes of 14 patients, and all cases $(100 \%)$ were HIV-infected males. Fifty percent (7/14) had been previously diagnosed with HIV infection and were under antiretroviral treatment at the primary hospital while the remaining cases were diagnosed with HIV infection at our clinic. Their mean age was $31 \pm 7$ (range, 22-43) years, and their mean CD4 count was 228 (range, 2-504) cells/ $\mu \mathrm{L}$. Testing of CSF obtained from lumbar puncture was conducted in $79 \%(11 / 14)$ and $91 \%(10 / 11)$ were RPR positive.

Table 1 shows the demographics and syphilis testing profiles of the patients in groups $1 \mathrm{~A}$ and $1 \mathrm{~B}$ (positive syphilis serology test). Sixty-four percent $(9 / 14)$ were diagnosed with neurosyphilis by a neurologist, including one patient (no.9) who was concurrently diagnosed with secondary syphilis. All patients in group $1 \mathrm{~A}$ had positive serum RPR and FTA-ABS IgG. Sixty-four percent (9/14) of subjects underwent EIA testing in serum, and all yielded positive results. Of these, all (14/14) had completed syphilis test (RPR, EIA, FTA-ABS lgG) for vitreous, and all vitreous samples yielded positive results. Seventy-one percent (10/14) underwent FTA-ABS IgM testing, yielding negative results in all vitreous samples. The median serum RPR titer among the 14 patients in group $1 \mathrm{~A}$ was $1: 256$ (range, 1:4-1:1024) compared with vitreous RPR titer of 1:16 (range, 1:1-1:1024). Twenty-nine percent (4/14) of the patients had RPR titers in vitreous equal to or higher than in serum.

\section{Clinical features and treatment outcomes of group $1 \mathrm{~A}$}

Of all 27 eyes from 14 patients, the most common ocular involvements of the definite SU group were chorioretinitis (25/27, $93 \%)$ and retinal vasculitis (25/27, 93\%). In bilateral cases, both eyes always disclosed the same anatomical type of uveitis, though asymmetry was present in some eyes. Uveitis was classified as posterior uveitis in 14 eyes (52\%) and panuveitis in the other 13 eyes (48\%). There were no cases of intermediate uveitis. Ninety-three percent of eyes (25/27) had initial BCVA worse than 20/60 and 41 percent $(11 / 27)$ had initial BCVA worse than 20/200. Patient 5, who presented with unilateral necrotizing chorioretinitis with exudative retinal detachment, underwent combined phacoemulsificationvitrectomy with silicone oil tamponade for repair of the detached retina when retinitis became inactive after intravenous penicillin $\mathrm{G}$. 


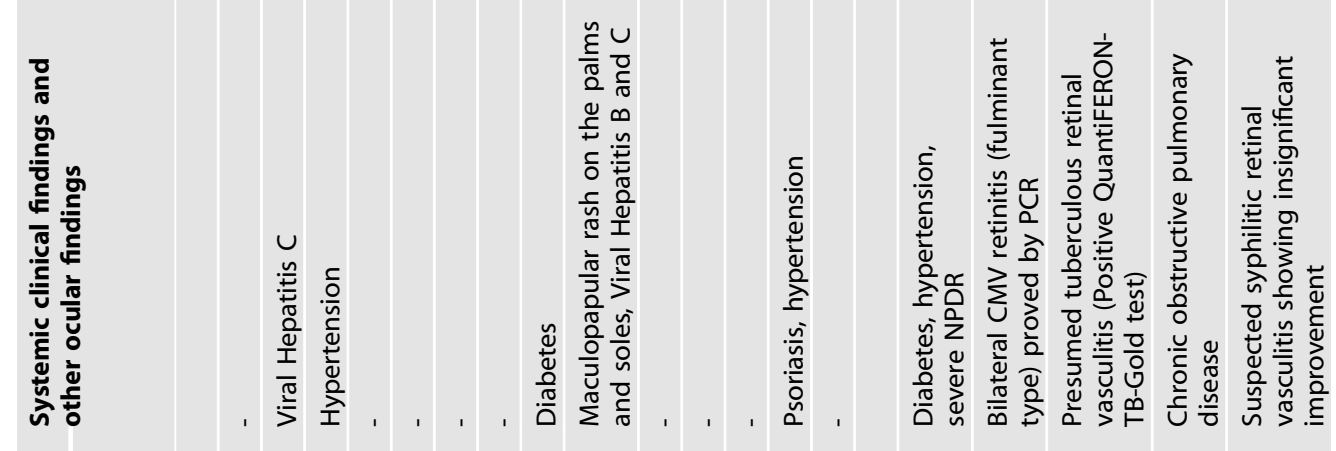



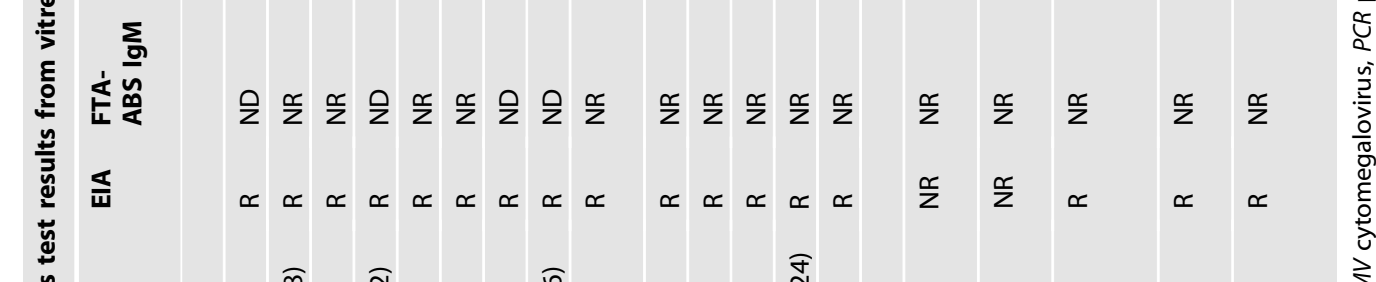

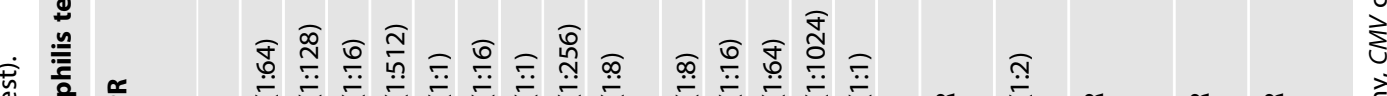

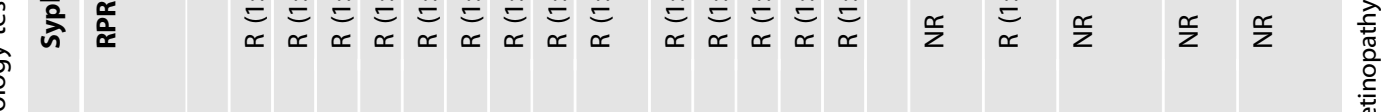

$\stackrel{\cong}{\frac{\varrho}{\bar{N}}}$

商

ลे

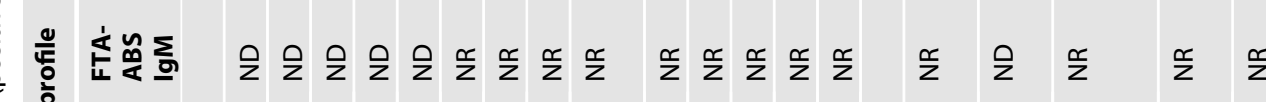

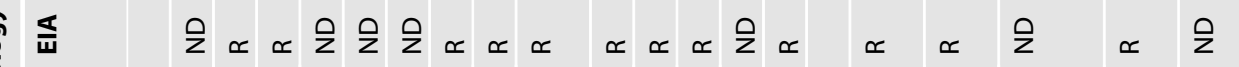

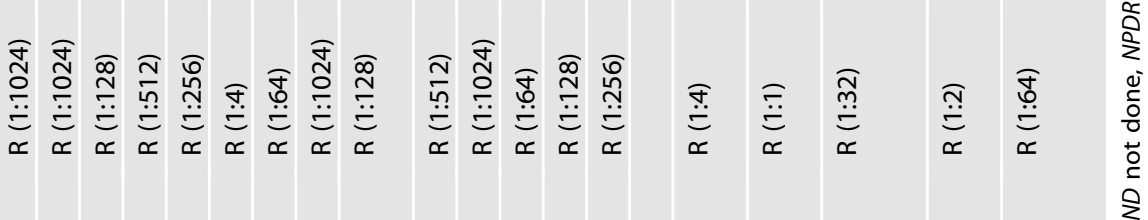

产

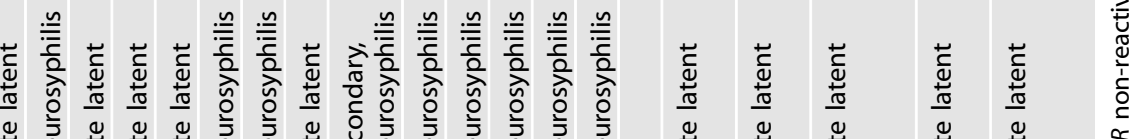

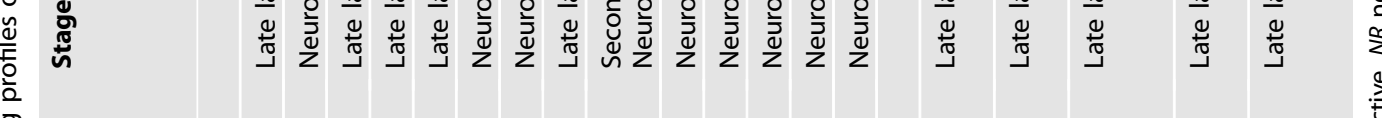

焉高至

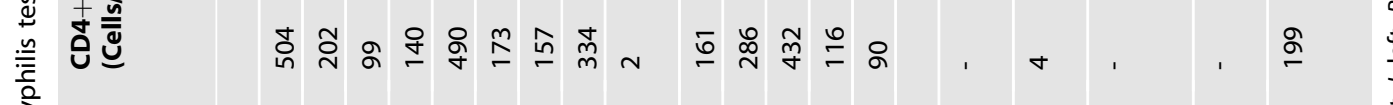

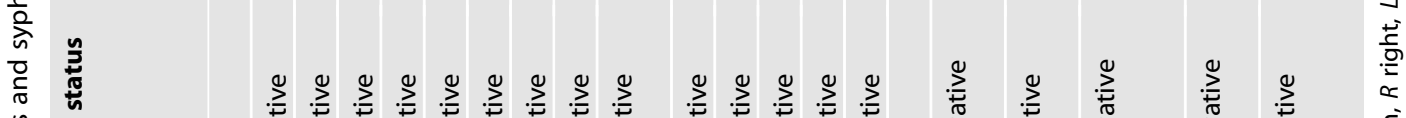

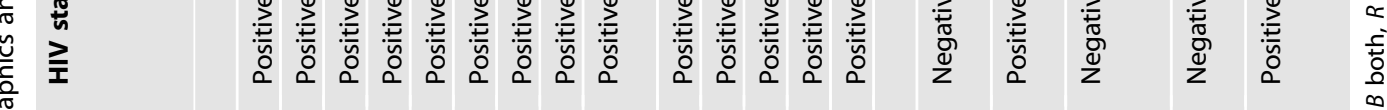

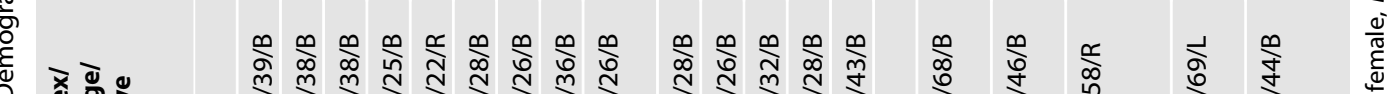

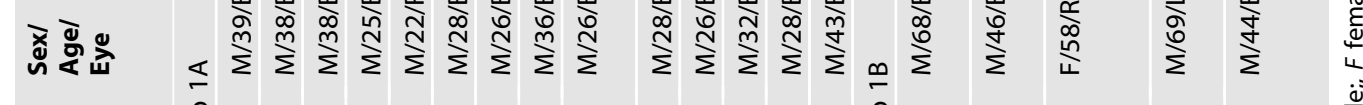
竞 




Twenty-four eyes (89\%) had OCT images and demonstrated disruption of the ellipsoid zone and focal disruption of the external limiting membrane. Twenty-five eyes (93\%) underwent FA and the most common angiographic features were retinal vascular leakage (predominately venous leakage or capillary leakage) (100\%), followed by optic disc leakage $(25 / 26,96 \%)$. Fig. $1 \mathrm{~A}-\mathrm{F}$ presents the imaging findings of cases 1 and 7 , which improved after treatment. There was no evidence of posterior placoid chorioretinitis in any case. Table 2 demonstrates the clinical characteristics and imaging findings of the patients in group $1 \mathrm{~A}$. 
Fig. 1 Multimodal imaging of selected cases in group 1A and group 1B. A-D show multimodal imaging of case 1 (group 1A). A SD-OCT image of the left eye shows disorganized outer nuclear and outer plexiform layer, focal defect of external limiting membrane, generalized ellipsoid zone disruption, and localized pigment epithelial detachment. B Two weeks after initiation of penicillin G, improvement was evident from the decrement of inner and outer retina irregularities in SD-OCT. There was no change in central choroidal thickness. C Mid-phase fluorescein angiogram of the left eye demonstrates perivascular and disc leakage. D Perifoveal leakage decreased after initiation of penicillin G. $\mathbf{E}$ and $\mathbf{F}$ show mid-phase fluorescein angiogram (FA) of case 7 (group 1A). $\mathbf{E}$ Though pre-treatment FA image is unclear due to the vitritis, it reveals disc leakage with diffuse venous leakage $\mathbf{F}$ Two weeks after the completion of intravenous ceftriaxone, the angiogram was clear, and there was significant reduction of leakage from optic disc and retinal veins. G-J show multimodal imaging of the patients in group 1 B. G and H Case 15 complained of floaters in the right eye with BCVA 20/20. The OCT image of the right eye shows relatively normal macula, and fluorescein angiogram reveals several leaking microaneurysms and capillary nonperfusion areas, which is characteristic of diabetic retinopathy. I and J Case 17 presented with unilateral retinal vasculitis which was focused in the macula (I). After a 2-week course of ceftriaxone monotherapy without visual improvement, oral prednisolone ( $1 \mathrm{mg} / \mathrm{kg}$ daily) was prescribed and showed good results in terms of visual gain and significant reduction of angiographic leakage at 1 week after corticosteroid treatment (J).

An equal number of patients were treated with penicillin $\mathrm{G}(7 /$ $14,50 \%)$ and ceftriaxone (7/14, 50\%). All patients showed significant clinical improvement in response to either penicillin $\mathrm{G}$ or ceftriaxone according to the aforementioned criteria. Topical prednisolone acetate $1 \%$ drop was started to control anterior chamber inflammation and did so successfully, and there was no use of systemic corticosteroids in any case. Visual improvement was achieved in all eyes at 1 month, at which point 63\% (17/27) experienced BCVA better than 20/60, and 85\% (23/27) experienced BCVA better than 20/200.

\section{Clinical features of group 1B}

Table 3 demonstrates the clinical characteristics, imaging findings, and clinical irrelevance to SU of the patients in group 1B. Patients 15,16 , and 17 were considered as not SU with different conditions (Fig. 1G-J) while patients 18 and 19 were indeterminate for SU. Lumbar puncture was performed only in cases 17 and 19, and CSF RPR results were negative. All patients were prescribed a 14-day course of either intravenous penicillin G (1/5) or ceftriaxone (4/5), and none showed significant clinical improvement according to the aforementioned criteria.

\section{Characteristics of groups $2 \mathrm{~A}$ and $2 \mathrm{~B}$}

Of all 38 participants, none was assigned to group 2A (negative syphilis serology and positive syphilis tests in vitreous), while 19 patients were classified into group $2 \mathrm{~B}$ (negative syphilis serology and negative syphilis tests in vitreous). Forty-seven percent $(9 / 19)$ were male and $16 \%(3 / 19)$ were HIV-infected. Their mean age was $53 \pm 18$ (range, 22-82) years. There were statistically significant differences relating to age $(P<0.001)$, sex $(P=0.001)$, and HIV infection $(P<0.001)$ between groups $1 \mathrm{~A}$ and $2 \mathrm{~B}$. Regarding group $2 \mathrm{~B}$, syphilis testing performed in vitreous samples of the controls were 100\% (19/19) for RPR, 100\% (19/19) for EIA, and 89\% (17/19) for FTA-ABS. All EIA and FTA-ABS rendered negative results. Most RPR yielded negative results with the exception of just $2(2 / 19$, $11 \%)$ controls who showed low titer RPR (1:2 and 1:4) in vitreous samples.

\section{Sensitivity and specificity of vitreous test for syphilis}

Syphilis serology tests (RPR, EIA, FTA-ABS lgG) were used as the diagnostic gold standard in our study. The most sensitive test for detecting syphilis antibodies in vitreous was EIA (90.9\%), followed by RPR (80.0\%) and FTA-ABS IgG (78.9\%) (See Supplementary information 3 for sensitivity, specificity, and kappa value for syphilis testing from vitreous specimens). EIA and FTA-ABS had the highest specificity, detecting $100 \%$ of the syphilis antibody. Regarding RPR titer in serum and vitreous, Spearman correlation showed that RPR titer in vitreous was significantly positively correlated with the RPR titer in serum $(R=0.78, P<0.001)$. Comparisons of the syphilis test results in vitreous with serum showed substantial agreement for RPR (kappa value, 0.69) and FTA-ABS (kappa value, 0.77) and almost perfect agreement for EIA (kappa value, 0.86).

\section{DISCUSSION}

While a serologic workup as the current standard for SU diagnosis is based either on traditional serologic testing or on reverse screening algorithms, such presumptive diagnoses can be problematic in some cases [1, 12-17]. Thus, there is a need to develop an accurate method to confirm diagnosis of SU besides PCR tests, which have limited sensitivity and availability [20-22]. The novel application of nontreponemal and treponemal antibody to confirm diagnosis of SU from vitreous samples has been previously pioneered by the current authors [14] and was affirmatively demonstrated by the present study. However, it is important, to emphasize that this method cannot replace PCR in terms of specificity to T. pallidum, and all patients testing positive for syphilis, whether or not they have SU, should seek specialist attention for extraocular diseases.

\section{Clinical features and patient classification}

A review of the literature suggests that characteristics of choroiditis and retinal vasculitis presenting in SU may be underreported, since most large case series have not provided comprehensive multimodal imaging analysis in exploring alterations in optic disc, retinal vessels, and outer retinal morphology [3, 5, 22, 27], while some small case series specifying a group of syphilitic chorioretinitis have clearly provided such data $[4,26,28]$. Our work with multimodal imaging found that chorioretinitis and retinal vasculitis were the most common manifestations in SU. Though SD-OCT findings in group $1 \mathrm{~A}$ disclosed alterations in external limiting membrane and ellipsoid zone as previously reported, there was no pattern of posterior placoid chorioretinopathy $[4,26,28]$. All patients in group $1 \mathrm{~A}$ fulfilled the criteria of clinical relevance and significant clinical improvement. Interestingly, they tested positive for all nontreponemal and treponemal antibody in vitreous specimens; therefore, we hypothesized that group $1 \mathrm{~A}$ should be determined as "definite $\mathrm{SU}^{\prime \prime}$ while patients in group 1B (not SU or indeterminate SU) presented with different features not typical of SU and had variable and fewer positive responses.

\section{Analysis of syphilis testing results and proposed algorithm table}

Sensitivity and specificity are useful summary measures for describing the diagnostic utility of a testing method. The current study, using syphilis serology tests as gold standard, demonstrated high sensitivity and specificity of syphilis test results from vitreous fluid. Also, RPR titer in vitreous showed a significantly positive correlation with RPR titer in serum. Interestingly, $29 \%$ of vitreous RPR in group $1 \mathrm{~A}$ revealed equal or higher titer than serum RPR. This is confirmatory evidence for the diagnosis of ocular syphilis. Unfortunately, group 1B comprised too few patients to enable evaluation of the interpretation. The present study also showed the lack of necessity for the antibody test in vitreous samples in patients with negative syphilis serology; however, we believe that this novel procedure could be beneficial in selected groups of patients as follows: (1) atypical clinical presentations of SU or unresponsiveness to the treatment [14-16]; (2) discordant or 




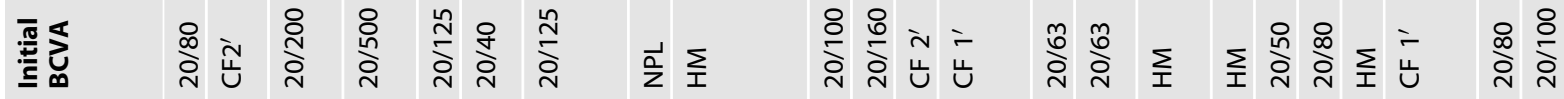

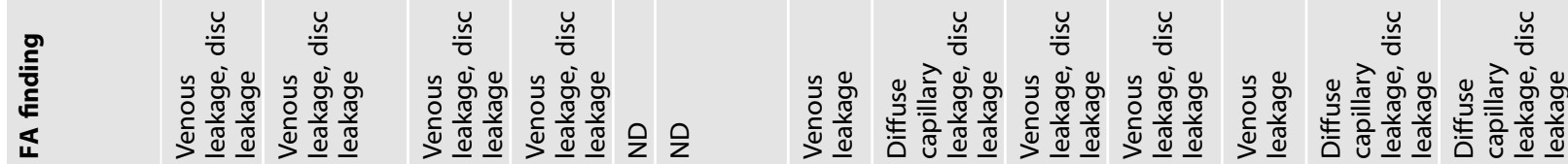

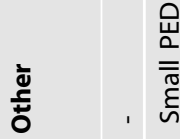

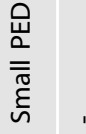
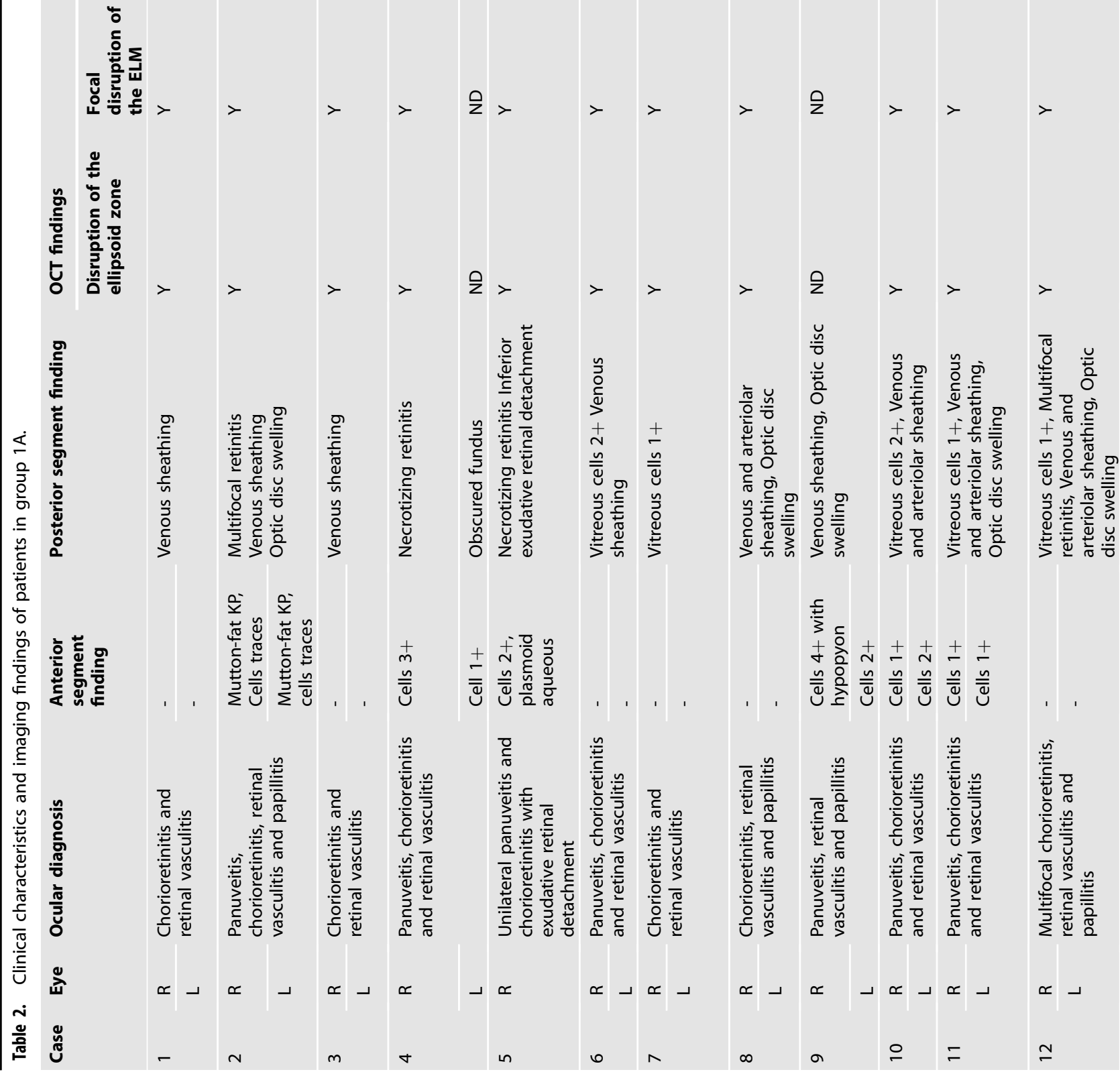
equivocal syphilis serology $[1,12,13]$; (3) medically complicated or immunocompromised patients where co-infection is possible $[15,17]$; and (4) patients referred with clinically suspected syphilis despite a negative serology, possibly due to the result of a laboratory error [7, 8]. Therefore, we presented the proposed algorithm according to our results (Table 4). Given the small number of tests included, all treponemal test results were classified as one testing for the algorithm. Furthermore, as we realized that the limited quantity of the vitreous specimens sometimes did not permit a full panel of testing, we recommended testing FTA-ABS IgG and RPR for a small volume of vitreous specimens (less than $0.3 \mathrm{ml}$ ), and full syphilis panel for sufficient volume of vitreous obtained (equal to or more than $0.3 \mathrm{ml}$ ). These tests should be performed sequentially, keeping RPR until last, as it requires more vitreous to be diluted in status of low antibody titer. We considered FTA-ABS IgG as a priority test in spite of its lower sensitivity compared with that of EIA, since higher testing numbers of FTA$A B S$ IgG demonstrate the strength of correlation. Communication between ophthalmologists and laboratory technicians is crucial before sending vitreous samples to the laboratory, since it is not conventional testing. We emphasize that this novel method should not be routinely performed, as it may become excessive.

This study had several limitations that are important to acknowledge. First, we used the results from serology as gold standard to analyze the sensitivity and specificity of syphilis testing from vitreous specimens; therefore, the negative results from vitreous specimens in group 1B may be due to the incompatible enzyme assay's not representing the true response. In addition, each test had limitations, including the potential for false-positive and false-negative results. Secondly, due to the nature of retina and uveitis tertiary care centers, selection bias may also have influenced results. Thirdly, the small number of patients included, as well as baseline discrepancies between groups, resulted in analyses that might be underpowered. Finally, the results are not necessarily generalizable to the population of patients with posterior uveitis given that all included cases in group $1 \mathrm{~A}$ were HIV-infected.

In conclusion, this study provided a novel approach and algorithm with the application of vitreous syphilis antibody as a supplementary test to confirm SU. In selected cases, as mentioned earlier, this method will increase the accuracy of presumptive diagnosis of SU that is conventionally made in clinical practice, and it will allow appropriate treatment to be commenced earlier. Further, it is applicable in all facilities but also requires communication between ophthalmologists and laboratory technicians in advance. However, further studies are required to evaluate other syphilis testing and confirm this observation.

\section{Summary}

What was known before

- Although in most cases, serological diagnosis and clinical presentations are sufficient to make a presumptive diagnosis of ocular syphilis, problems with diagnosis and treatment may occur in selected uveitis patients if they have discordant or equivocal syphilis serology, atypical clinical presentation, or, in immunocompromised patients, possible co-infection.

- There is a need to develop an accurate method to confirm the diagnosis of syphilitic uveitis besides PCR tests, which have limited sensitivity and availability.

What this study adds

- The testing of nontreponemal and treponemal antibodies in vitreous can be applicable to improve the accuracy of the presumptive diagnosis of syphilitic uveitis, and could be beneficial in selected groups of patients 


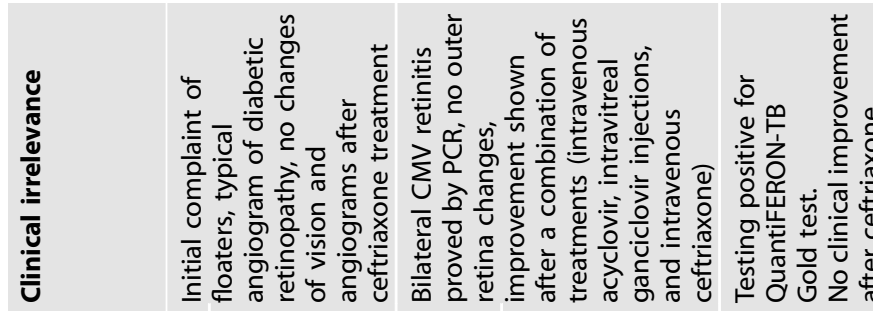

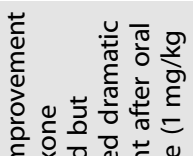

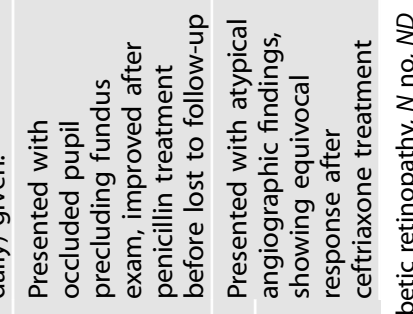

ปัँ



오ำ

$\sum$

유 గి

$\frac{\square}{3}$

유 유

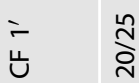

กิ

๗ัก



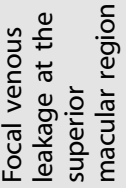

之

$\sum$
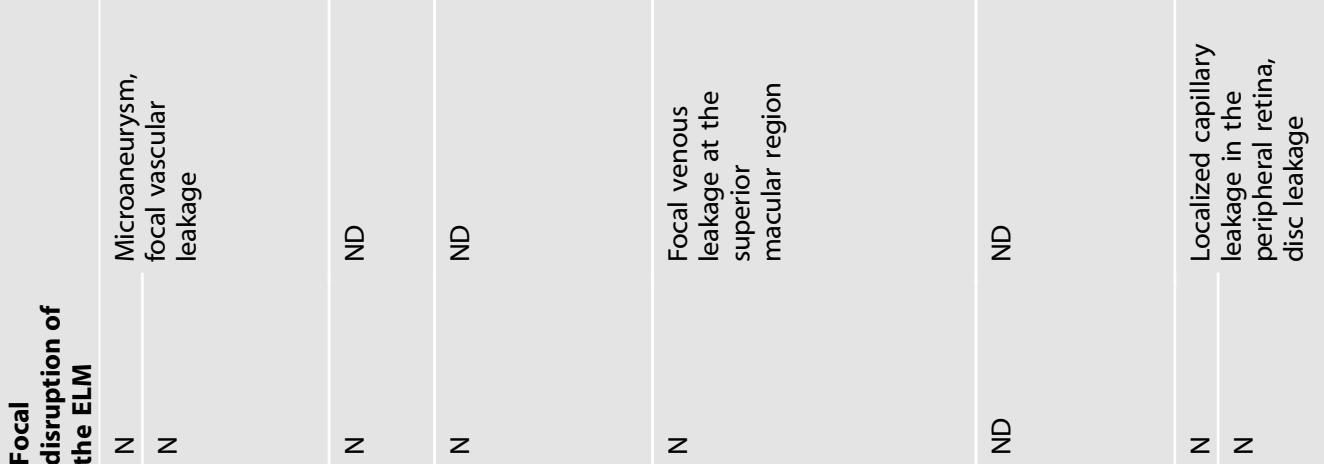

只

ㅇㅝㅀㅀㅎㅎㅇ

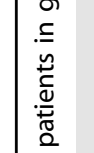

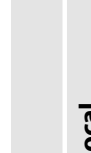

岳

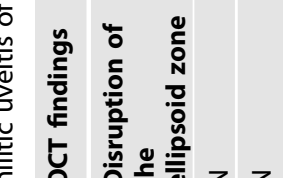

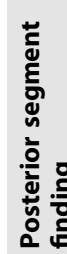

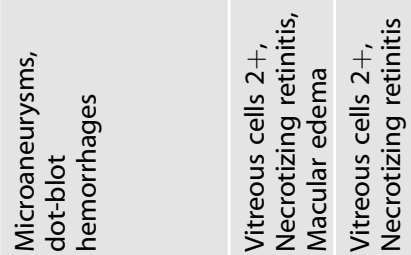

z

₹

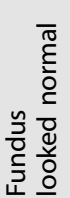

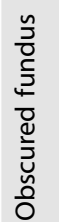

z z

(

듬

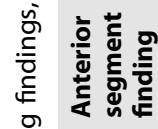

$\begin{array}{ll}+ & + \\ \stackrel{n}{m} & \cong\end{array}$

$\frac{ \pm}{\bar{\Xi}}$

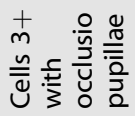
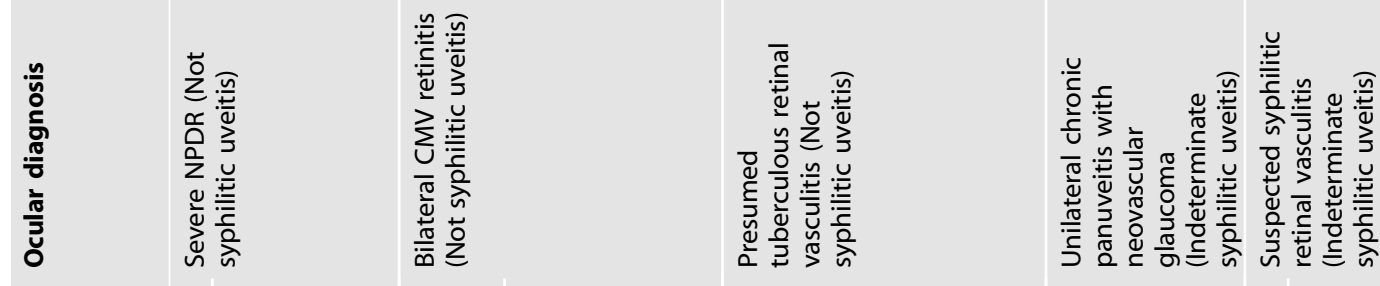

斊
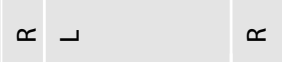

$\because$

I

$\stackrel{\infty}{-}$

$\simeq$

문

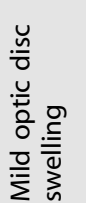


Table 4. Interpretation of syphilis test results from vitreous.

\begin{tabular}{|c|c|c|}
\hline Treponemal tests (EIA or FTA-ABS) & Nontreponemal tests (RPR) & Likely presentation \\
\hline+ & + & Definite syphilitic uveitis \\
\hline+ & - & Probable syphilitic uveitis \\
\hline \multirow[t]{2}{*}{-} & + & Vitreous RPR titer $\geq$ serum titer: probable syphilitic uveitis ${ }^{a}$ \\
\hline & & Vitreous RPR titer < serum titer: possible syphilitic uveitis ${ }^{a}$ \\
\hline- & - & Not syphilitic uveitis ${ }^{a}$ \\
\hline
\end{tabular}

${ }^{a}$ Other treponemal tests can be considered to confirm the diagnosis.

\section{REFERENCES}

1. Tuddenham S, Obeng C, Ghanem KG. Neurosyphilis and ophthalmic syphilis in persons with negative rapid plasma reagin and positive treponemal antibody test results. Sex Transm Dis. 2015;42:347-9.

2. Davis JL. Ocular syphilis. Curr Opin Ophthalmol. 2014;25:513-8.

3. Bollemeijer JG, Wieringa WG, Missotten TO, Meenken I, ten Dam-van NH, Rothova A. et al. Clinical manifestations and outcome of syphilitic uveitis. Investig Ophthalmol Vis Sci. 2016;57:404-11.

4. Pichi F, Ciardella AP, Cunningham ET Jr, Morara M, Veronese C, Jumper JM, et al. Spectral domain optical coherence tomography findings in patients with acute syphilitic posterior placoid chorioretinopathy. Retina 2014;34:373-84.

5. Moradi A, Salek S, Daniel E, Gangaputra S, Ostheimer TA, Burkholder BM, et al. Clinical features and incidence rates of ocular complications in patients with ocular syphilis. Am J Ophthalmol. 2015;159:334-43 e1.

6. Tran TH, Cassoux N, Bodaghi B, Fardeau C, Caumes E, Lehoang P. Syphilitic uveitis in patients infected with human immunodeficiency virus. Graefes Arch Clin Exp Ophthalmol. 2005;243:863-9.

7. Katz AR, Komeya AY, Tomas JE. False-negative syphilis treponemal enzyme immunoassay results in an HIV-infected case-patient. Int J STD AIDS. 2017;28:735-7.

8. Blum L, Bachmeyer C, Caumes E. Seronegative secondary syphilis in an HIVinfected patient. Clin Exp Dermatol. 2005;30:158-9.

9. Ortega KL, Rezende NP, Magalhaes MH. Diagnosing secondary syphilis in a patient with HIV. Br J Oral Maxillofac Surg. 2009;47:169-70.

10. Geusau A, Kittler H, Hein U, Dangl-Erlach E, Stingl G, Tschachler E. Biological falsepositive tests comprise a high proportion of Venereal Disease Research Laboratory reactions in an analysis of 300,000 sera. Int J STD AIDS. 2005;16:722-6.

11. Lynn WA, Lightman S. Syphilis and HIV: a dangerous combination. Lancet Infect Dis. 2004;4:456-66.

12. Smith JL, Singer JA, Moore MB Jr, Yobs AR. Seronegative ocular and neurosyphilis. Am J Ophthalmol. 1965:59:753-62.

13. Tamesis RR, Foster CS. Ocular syphilis. Ophthalmology 1990;97:1281-7.

14. Silpa-Archa S, Preble JM, Foster CS. Vitreous treponemal antibody as a supplementary test to serology for the confirmation of syphilitic chorioretinitis. Retin Cases Brief Rep. 2020;14:166-9.

15. Troutbeck R, Chhabra R, Jones NP. Polymerase chain reaction testing of vitreous in atypical ocular syphilis. Ocul Immunol Inflamm. 2013;21:227-30.

16. Ormaechea MS, Hassan M, Nguyen QD, Schlaen A. Acute syphilitic posterior placoid chorioretinopathy: an infectious or autoimmune disease? Am J Ophthalmol Case Rep. 2019;14:70-3.

17. Amaratunge BC, Camuglia JE, Hall AJ. Syphilitic uveitis: a review of clinical manifestations and treatment outcomes of syphilitic uveitis in human immunodeficiency virus-positive and negative patients. Clin Exp Ophthalmol. 2010;38:68-74.

18. Rajan MS, Pantelidis P, Tong CY, French GL, Graham EM, Stanford MR. Diagnosis of Treponema pallidum in vitreous samples using real time polymerase chain reaction. Br J Ophthalmol. 2006;90:647-8.

19. Booth J, Rodger A, Singh J, Alexander S, Hopkins S. Syphilitic panuveitis with retinal necrosis in an HIV positive man confirmed by Treponema pallidum PCR. J Infect. 2009;59:373-5.

20. Grange PA, Gressier L, Dion PL, Farhi D, Benhaddou N, Gerhardt P, et al. Evaluation of a PCR test for detection of treponema pallidum in swabs and blood. J Clin Microbiol. 2012;50:546-52.

21. Wang C, Cheng Y, Liu B, Wang Y, Gong W, Qian Y, et al. Sensitive detection of Treponema pallidum DNA from the whole blood of patients with syphilis by the nested PCR assay. Emerg Microbes Infect. 2018;7:83.
22. Hoogewoud F, Frumholtz L, Loubet $P$, Charlier C, Blanche $P$, Lebeaux D, et al. Prognostic factors in syphilitic uveitis. Ophthalmology. 2017;124:1808-16.

23. Jabs DA, Nussenblatt RB, Rosenbaum JT, Standardization of Uveitis Nomenclature Working G. Standardization of uveitis nomenclature for reporting clinical data. Results of the First International Workshop. Am J Ophthalmol. 2005;140:509-16.

24. Buitrago-Garcia D, Marti-Carvajal AJ, Jimenez A, Conterno LO, Pardo R. Antibiotic therapy for adults with neurosyphilis. Cochrane Database Syst Rev. 2019;5: CD011399.

25. Workowski KA, Bolan GA, Centers for Disease C, Prevention. Sexually transmitted diseases treatment guidelines, 2015. MMWR Recomm Rep. 2015;64:1-137. RR-03

26. Eandi CM, Neri P, Adelman RA, Yannuzzi LA, Cunningham ET Jr, International Syphilis Study G. Acute syphilitic posterior placoid chorioretinitis: report of a case series and comprehensive review of the literature. Retina. 2012;32:1915-41.

27. Mathew RG, Goh BT, Westcott MC. British Ocular Syphilis Study (BOSS): 2-year national surveillance study of intraocular inflammation secondary to ocular syphilis. Investig Ophthalmol Vis Sci. 2014;55:5394-400.

28. Zhu J, Jiang $Y$, Shi $Y$, Zheng $B, X u Z$, Jia W. Clinical manifestations and treatment outcomes of syphilitic uveitis in HIV-negative patients in China: a retrospective case study. Medicine. 2017;96:e8376.

\section{ACKNOWLEDGEMENTS}

The authors would like to thank Dr. Mongkol Tadarati and Dr. Jirawut Limwattanayingyong for referring the study participants, Dr. Jaruda Kobkitjaroen for the analysis of syphilitic testing, Mrs. Chananuch Plang-ngern, together with her colleagues, for their laboratory data support, and Mr. John Flanagan for English editing.

\section{AUTHOR CONTRIBUTIONS}

SS conceived the study, participated in design and coordination, performed data interpretation, and drafted and edited the article. TH participated in coordination and data collection. CSF edited the article. All authors read and approved the final version of the article.

\section{COMPETING INTERESTS}

The authors declare no competing interests.

\section{ADDITIONAL INFORMATION}

Supplementary information The online version contains supplementary material available at https://doi.org/10.1038/s41433-021-01902-6.

Correspondence and requests for materials should be addressed to Sukhum Silpaarcha.

Reprints and permission information is available at http://www.nature.com/ reprints

Publisher's note Springer Nature remains neutral with regard to jurisdictional claims in published maps and institutional affiliations. 